\title{
Colgajo radial: experiencia del equipo de Cirugía Plástica de la Universidad de Chile*
}

\author{
Drs. PATRICIO ANDRADES C. ${ }^{1}$, MARÍA ELSA CALDERÓN G. ${ }^{1}$, STEFAN DANILLA E. ${ }^{1}$, \\ SUSANA BENÍTEZ S. ${ }^{1}$, CRISTIAN ERAZO C. ${ }^{1}$, SERGIO SEPÚLVEDA. ${ }^{1}$ \\ 1 Departamento de Cirugía, Hospital Clínico Universidad de Chile. \\ Santiago, Chile.
}

\begin{abstract}
Radial forearm flaps. Experience in ten patients

Background: Radial forearm flap is extraordinarily versatile thanks to its irrigation by the radial artery and its minor pedicles. It provides assorted alternatives for the reconstruction of proximal or distal defects of the arm and can be used as a free flap in head, neck, posterior trunk region, lower limb, esophagus and penis. It allows the incorporation of bone, tendons, nerves and muscle for complex lesion repair. Aim: To report our experience with radial forearm flap. Patients and Methods: Ten patients aged 20 to 65 years (four women) are reported. The lesions repaired were traumatic in four, infectious in three, secondary to tumors in two (a squamous intra oral adenocarcinoma in both patients) and vascular in one patient. Results: No patient had a partial or total loss of the flap. Five patients required complementary dermo epidermic grafts in a second intervention. Mean hospital stay was 30 days. All patients reported a favorable degree of satisfaction with the procedure. Conclusions: Radial forearm flaps are a good alternative for the repair of a great variety of lesions.

Key words: Radial flap, cutaneous lesion, fasciocutaneous flap.
\end{abstract}

\section{Resumen}

El colgajo radial, es un colgajo fasciocutáneo tipo II según Mathes y Nahai, de gran versatilidad gracias a su irrigación principal por la arteria radial y a sus pedículos menores, ofrece una amplia variedad de alternativas frente a la reconstrucción no sólo de defectos proximales o distales del brazo, sino que también es posible utilizarlo como colgajo libre en defectos de cabeza y cuello, porción posterior del tronco, extremidad inferior y reconstrucción de esófago y pene. Permite incorporar hueso, tendones, nervio y músculo para reparación de lesiones complejas, pudiéndose utilizar con flujo directo o flujo retrógrado. En el presente artículo, mostramos la experiencia adquirida por el equipo de Cirugía Plástica y Reconstructiva del Hospital Clínico de la Universidad de Chile en la utilización del colgajo radial, como alternativa de cobertura frente a diversos tipos de defectos. En nuestros resultados, el 100\% de los pacientes evolucionó favorablemente, sin pérdidas parciales ni totales del colgajo; sólo algunos debieron recibir injertos dermo-epidérmicos (IDE) complementarios en un segundo tiempo, sin ningún otro procedimiento quirúrgico posterior al alta.

Palabras clave: Colgajo radial, colgajo fasciocutáneo.

*Recibido el 18 de abril de 2011 y aceptado para publicación el 16 de junio de 2011.

Correspondencia: Dr. Patricio Andrades C.

Santos Dumont No 999, Santiago, Chile.

pandrades@u.uchile.cl 


\section{Introducción}

El colgajo radial fue realizado por primera vez el 29 de Marzo de 1979 como colgajo libre para cobertura de mano, en el Departamento de Cirugía del Hospital General de Shenyang en China. La primera publicación acerca de este colgajo apareció en el "National Medical Journal of China" en 1981. Hecho que lo mantuvo aislado del mundo occidental durante casi 16 años en que recién se logra publicar "traducido" en el British Journal of Plastic Surgery del año 1997'; a partir de ese momento se da a conocer a nivel mundial con una rápida aceptación, convirtiéndose en el "caballito de batalla” utilizado por distintos especialistas para la reconstrucción de múltiples y variados defectos. En la actualidad es ampliamente conocido como "colgajo chino", con tal grado de popularidad que al cumplir los 25 años de ser presentado al mundo científico, fue realizado en la misma ciudad de Shenyang en China, un Simposio de carácter mundial que se centró únicamente en el desarrollo y los logros del colgajo radial obtenidos a lo largo de su historia. Ese mismo año 2006 el Departamento de Cirugía Plástica del Hospital Militar de Shenyang, publica el libro con la "Historia del Colgajo Radial” en el que se recopilaron anécdotas, modificaciones y evolución de la técnica quirúrgica, estadística y datos científicos descritos desde su inicio hasta nuestros días.

Claramente podemos ver que desde su introducción, el Colgajo Radial ha demostrado ser una herramienta tremendamente versátil, que nos permite incorporar piel, huesos, tendones, nervio y músculo para reparar lesiones complejas, con flujo directo, retrógrado o en forma libre dependiendo del defecto a cubrir, permitiéndonos así una amplia gama de alternativas en reconstrucción. Dada la relevancia de este gran colgajo, que lo ha mantenido en vigencia hasta el día de hoy, motivados por los pacientes que se vieron beneficiados con su utilización, los satisfactorios resultados obtenidos y a la conmemoración de los 30 años desde su creación; hemos decidido destacar su aniversario en la literatura nacional, presentando con casos propios, la experiencia en la utilización del colgajo radial como alternativa de cobertura en reconstrucción de distintos tipos de defectos, a cargo del equipo de Cirugía Plástica del Hospital Clínico de la Universidad de Chile.

\section{Material y Método}

El siguiente es un estudio longitudinal, descriptivo y de seguimiento en el que se presenta la anatomía funcional de la arteria radial, la técnica quirúrgica para el levantamiento del colgajo radial desde la zona dadora hasta la colocación en la zona receptora, y la presentación de diez casos operados por el equipo de Cirugía Plástica y Reconstructiva del HCUCH entre el período 1999-2010.

\section{Anatomía funcional de la arteria radial}

La arteria radial, continuación de la arteria braquial, corre lateral al septum intermuscular, por debajo de los músculos braquioradial y flexor radial del carpo, separando los compartimientos de los músculos flexores y extensores del antebrazo. Desde su origen a nivel del pliegue del brazo y hasta 1-3 $\mathrm{cm}$ proximal a la apófisis estiloides, emite una serie de ramos perforantes que a través de las fascias profundas, septales y septocutáneas van a irrigar a los músculos, huesos y 3/4 partes la piel del antebrazo ${ }^{2}$.

Con un total aproximado de 9 a 17 ramos perforantes fasciocutáneos, se distribuyen a lo largo de la arteria radial con una frecuencia de cada 0,5 a $1,5 \mathrm{~cm}$, pero son divididos principalmente en dos grupos: el pedículo proximal que se estima corresponde al $61,7 \%$ de los perforantes, y el pedículo distal con un $17,6 \%$ de los ramos; sin embargo, en un artículo reciente, basado en el estudio de los vasos perforantes de la arteria radial realizado en 26 cadáveres $^{2,3}$, se ha identificado que a nivel distal destaca un ramo perforante "constante y de mayor calibre" en el $100 \%$ de los casos estudiados, que mide entre 0,5 y 0,8 mm de diámetro y que está a 2 cm de la apófisis estiloides; razón que actualmente lo convierte en uno de los fundamentos para el estudio clínico a nivel experimental del "colgajo local de ramos perforantes de la arterial radial”, en donde se utilizan sólo estos vasos perforantes, manteniendo la indemnidad de la arteria radial, para la reconstrucción de determinados tipos de defectos ${ }^{3-5}$.

\section{Técnica quirúrgica}

Como podemos apreciar, la estructura anatómica de la arteria radial nos permite escoger el realizar un colgajo radial de pedículo proximal, de pedículo distal, o un colgajo libre que a su vez puede ser fasciocutáneo u osteocutáneo, dependiendo del defecto que necesitemos cubrir. Se debe diseñar la isla cutánea requerida que puede ser bilobulada, longitudinal, en omega o rectangular, con un máximo aceptado de 20 x $10 \mathrm{~cm}$ como zona dadora y un largo del pedículo según necesidad. El colgajo radial a pedículo proximal nos permite abarcar tanto la zona del codo como de la fosa antecubital. El colgajo radial de pedículo distal llega a cubrir dorso y palma de la mano, así como también el pulgar y la $1^{\mathrm{a}}$ y $2^{\mathrm{a}}$ falange de los dedos índice al meñique. Para los colgajos libres, ya sea este fasciocutáneo u osteocutáneo, las zonas receptoras pueden ser muy variadas; se utiliza principalmente en casos de cobertura de defectos de 
cabeza y cuello (partes blandas, piso de boca, lengua, faringe, esófago) o en reconstrucción de pene. Aquellas lesiones que requieran además soporte óseo disponen del colgajo radial osteocutáneo, como por ejemplo en reconstrucción de maxilar superior o mandíbula (Figura 1).

La técnica clásica del levantamiento del colgajo radial consiste en realizar inicialmente la isquemia del antebrazo mediante un torniquete neumático, diseñar el colgajo adecuado y realizar la incisión en piel y tejido celular subcutáneo, teniendo especial precaución con los vasos perforantes y de identificar y preservar la rama sensitiva del nervio radial que debiera encontrarse lateral al tendón del músculo braquioradial. Una vez identificada la arteria radial y sus venas acompañantes (generalmente la cefálica) se individualiza el pedículo con la isla cutánea hasta el punto pivote específico: si se trata de un colgajo de pedículo proximal éste será a nivel del origen de la arteria radial en la fosa antecubital; si se trata de un colgajo de pedículo distal el punto pivote será a nivel de la apófisis estiloides en donde se percibe el pulso radial. En el caso de los colgajos libres se seccionan los vasos que posteriormente serán anastomosados bajo técnica microquirúrgica al lecho receptor.

Una vez comprobados la viabilidad del colgajo y con un adecuado control de hemostasia posterior al retiro del torniquete, se coloca el colgajo en la zona receptora cuidando siempre de no comprimir o torcer el pedículo vascular.

En el caso del colgajo libre osteocutáneo, tomando idealmente como zona dadora al brazo "no dominante”, se sigue el mismo procedimiento pero tratando de preservar la fascia, visualizar y mantener los vasos perforantes aferentes al músculo braquioradial y al hueso radial, la sección ósea se realiza con un corte en bisel con un máximo de $10 \mathrm{~cm}$ de largo y de no más del $40 \%$ de la circunferencia del hueso radial. La colocación de placas de fijación ósea a la zona dadora es la conducta con la que actualmente se previene en forma adecuada la fractura patológica del hueso radial ${ }^{6,7}$.

\section{Pacientes}

Se describen a continuación un total de 10 pacientes, de los cuales 4 son mujeres y 6 son hombres, entre los 20 y los 65 años de edad, con una media de 58 años.

Dentro de las patologías con indicación quirúrgica de reconstrucción tenemos: cuatro casos de lesiones traumáticas (40\%), tres de origen infeccioso (30\%), dos de origen neoplásico: adenocarcinoma escamoso intraoral en ambas (20\%), y un paciente con una lesión de origen vascular (10\%).

De las técnicas quirúrgicas de cobertura utilizadas con el colgajo radial; se realizaron cinco colgajos radiales a pedículo distal (50\%), cuatro colgajos radiales libres fasciocutáneos (40\%) y un colgajo radial libre osteocutáneo (10\%) (Tabla 1).

\section{Resultados}

Del total de pacientes atendidos que debieron recibir al Colgajo Radial en alguna de sus formas, ninguno de ellos tuvo alguna pérdida parcial o total del mismo, debieron realizarse IDE complementarios en

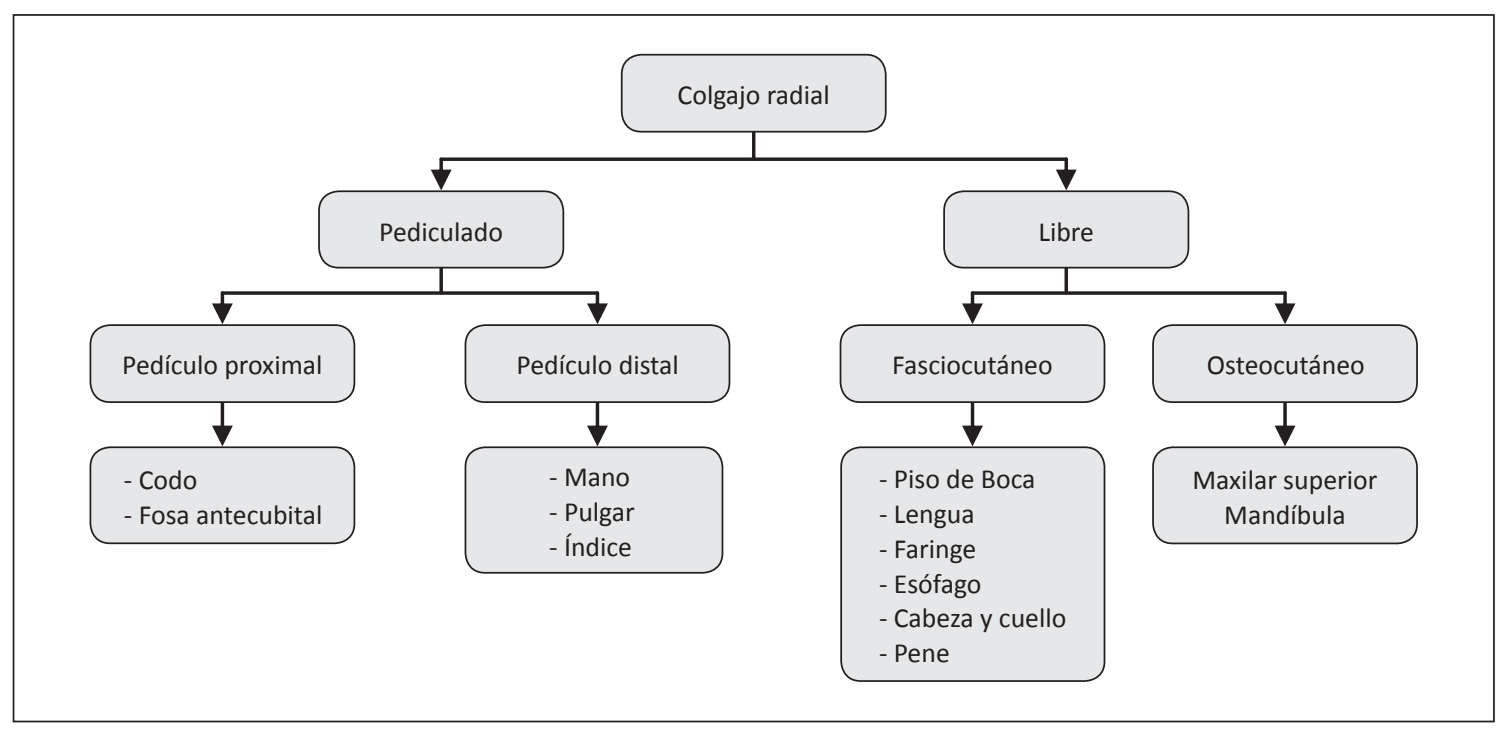

Figura 1. Organigrama de técnicas quirúrgicas de cobertura utilizando el colgajo radial. 
Tabla 1. Diagnósticos y tratamiento quirúrgico efectuado en los pacientes con reconstrucción a través de distintos tipos de Colgajos Radiales

\begin{tabular}{|c|c|c|c|c|c|}
\hline $\mathbf{n}$ & Sexo & Edad & Diagnóstico & Cirugía & Evolución \\
\hline 1 & $\mathrm{~F}$ & 58 & Fasceitis necrotizante mano y antebrazo der. & Colgajo radial libre & 12 años - alta \\
\hline 2 & M & 42 & Atrición mano der. & Colgajo radial libre & 8 años - alta \\
\hline 3 & M & 59 & Malformación vascular índice der. & Colgajo radial pedículo distal & 6 años - alta \\
\hline 4 & M & 55 & Fasceitis necrotizante dorso de mano & Colgajo radial pedículo distal & 3 años - en control \\
\hline 5 & $\mathrm{~F}$ & 43 & Fasceitis necrotizante mano y antebrazo izq. & Colgajo radial pedículo distal & $\begin{array}{l}1 \text { año, } 5 \text { meses - } \\
\text { en control }\end{array}$ \\
\hline 6 & $\mathrm{~F}$ & 58 & Ca. Escamoso intraoral & Colgajo radial libre & $\begin{array}{l}2 \text { años, } 4 \text { meses - } \\
\text { en control }\end{array}$ \\
\hline 7 & M & 63 & Fx. Expuesta de $2^{\text {do }}$ y $3^{\text {er }}$ metacarpiano & Colgajo radial pedículo distal & 1 año - en control \\
\hline 8 & M & 20 & Defecto dorso de mano por arma de fuego & Colgajo radial pedículo distal & $\begin{array}{l}1 \text { año, } 2 \text { meses - } \\
\text { en control }\end{array}$ \\
\hline 9 & M & 67 & Fístula cráneo-oftalmo-naso-cutánea & Colgajo radial libre & $\begin{array}{l}8 \text { meses - en } \\
\text { control }\end{array}$ \\
\hline 10 & F & 65 & Ca. Escamoso intraoral y mandibular & Colgajo radial libre osteocutáneo & $\begin{array}{l}7 \text { meses - con Qt } \\
\text { y Rt en control }\end{array}$ \\
\hline
\end{tabular}

un segundo tiempo en cinco de los diez pacientes, pero posterior al alta, ninguno de ellos presentó complicaciones significativas ni requerimiento de un nuevo procedimiento quirúrgico posterior al alta.

El tiempo promedio de estadía hospitalaria fue de 30 días. Se describe un período de seguimiento establecido por protocolo, que va desde el postoperatorio inmediato hasta el alta definitiva a los cinco años de la cirugía. Del total de pacientes, la evolución ha sido satisfactoria en todos los casos: tres de ellos ya superaron el período de seguimiento anual y se encuentran de alta definitiva, sin secuelas funcionales y con una mínima repercusión estética, los otros siete pacientes aún se encuentran en postoperatorio normal alejado y tardío, en controles trimestrales aquellos con menos de un año de la cirugía y en controles anuales los que se encuentran entre el $1^{\text {er }}$ y $5^{\text {to }}$ año del postoperatorio (Tabla 1). El grado de percepción/satisfacción por equipo médico y pacientes post procedimiento es favorable en todos los casos, con notables mejoras en los hábitos y la calidad de vida de los pacientes.

A continuación, presentamos los casos clínicos más representativos, del grupo de diez pacientes atendidos en nuestra unidad de Cirugía Plástica y Reconstructiva, explicando la resolución quirúrgica empleada, junto a la evolución durante el postoperatorio inmediato y alejado:

Caso $N^{o}$ 1. Colgajo radial de pedículo distal

Paciente masculino 59 años, portador de mal- formación vascular índice derecho de larga data. Consulta el año 2005 por dolor, fragilidad capilar, sangrados espontáneos intermitentes que en una oportunidad requirió hospitalización por anemia aguda. Con incapacidad laboral hasta el momento de la cirugía.

Se realiza una reconstrucción con colgajo radial de pedículo distal, de la cual evoluciona en postoperatorio favorablemente, requirió colocación de un IDE complementario < 1\% durante la misma hospitalización, sin complicaciones posteriores, recupera funcionalidad de la extremidad en un $100 \%$. Actualmente, a los cinco años de la cirugía se encuentra de alta (Figura 2).

\section{Caso $N^{o}$ 2. Colgajo libre fasciocutáneo}

Paciente masculino de 50 años, el año 1994 sufre accidentalmente corte facial extenso por sierra de banco, con gran defecto parietal, frontal, nasal y labial superior, con fractura naso-etmoidal bilateral y fractura fronto-orbitaria. Se realiza reconstrucción de las paredes de la órbita con placas + injertos óseos en defecto fronto-parietal. Con adecuado cierre de partes blandas, evoluciona al año siguiente con fístula cráneo-naso-oftalmocutánea, con débito purulento activo, que se mantiene en el tiempo pese a distintos intentos de manejo local, se decide reconstrucción con colgajo radial libre fasciocutáneo el año 2010. Evolución satisfactoria en los primeros ocho meses del postoperatorio (Figura 3). 


\section{Caso $N^{\circ}$ 3. Colgajo libre osteocutáneo}

Paciente femenina de 75 años, portadora de un carcinoma de células escamosas a nivel de maxilar inferior, consulta el año 2010 por dolor, aumento de volumen sobre arcada dentaria inferior con sangrado espontáneo, dificultad para el habla y la alimentación. Con estudio de diseminación negativo,
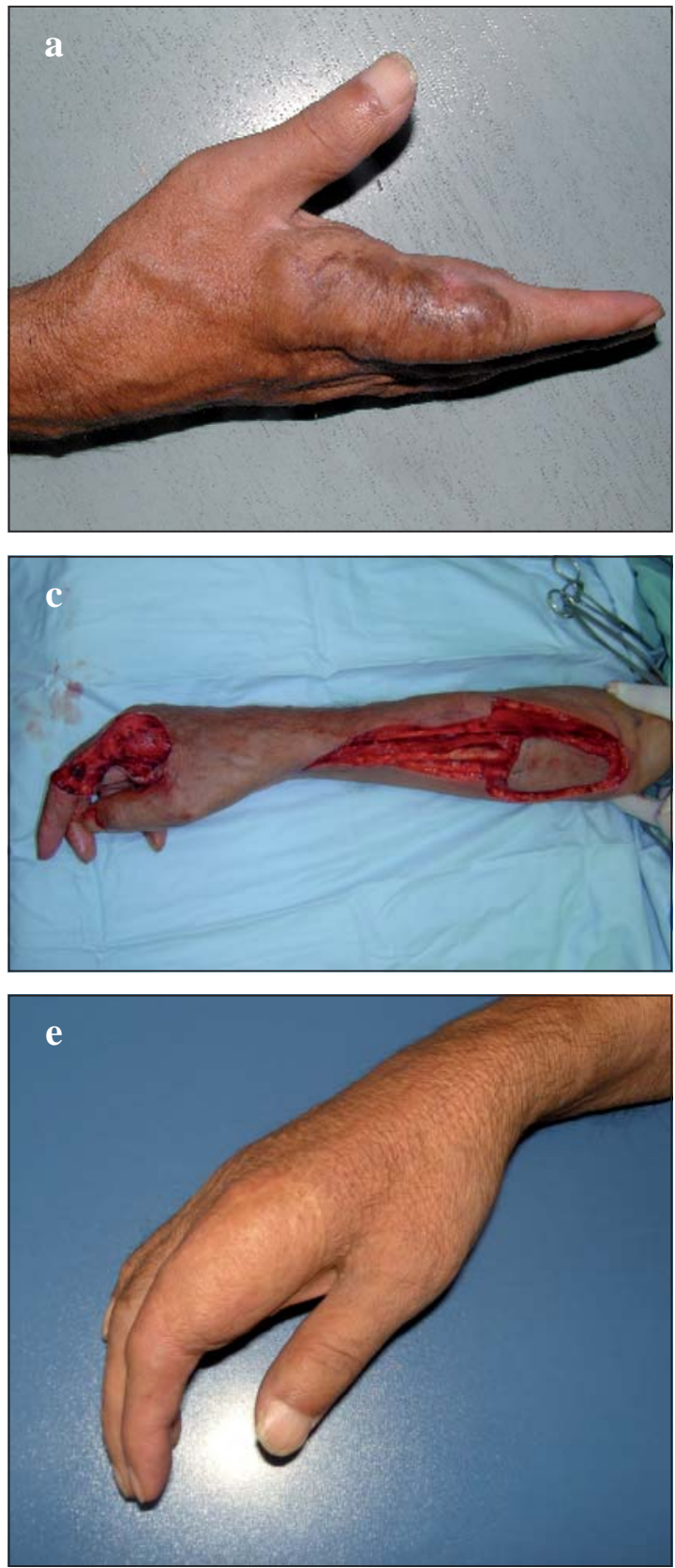

Figura 2. a) malformación vascular; b) arteriografia preoperatoria; c y d) intraoperatorio; e y f) control postoperatorio a los 5 años.

se plantea la resección oncológica del tumor, con vaciamiento radical cervical y reconstrucción con colgajo libre osteocutáneo. Durante la cirugía se trabajó a dos equipos en conjunto con los especialistas de cabeza y cuello. Se realizó el levantamiento del colgajo libre osteocutáneo radial, con colocación de una placa de osteosíntesis e inmovilización
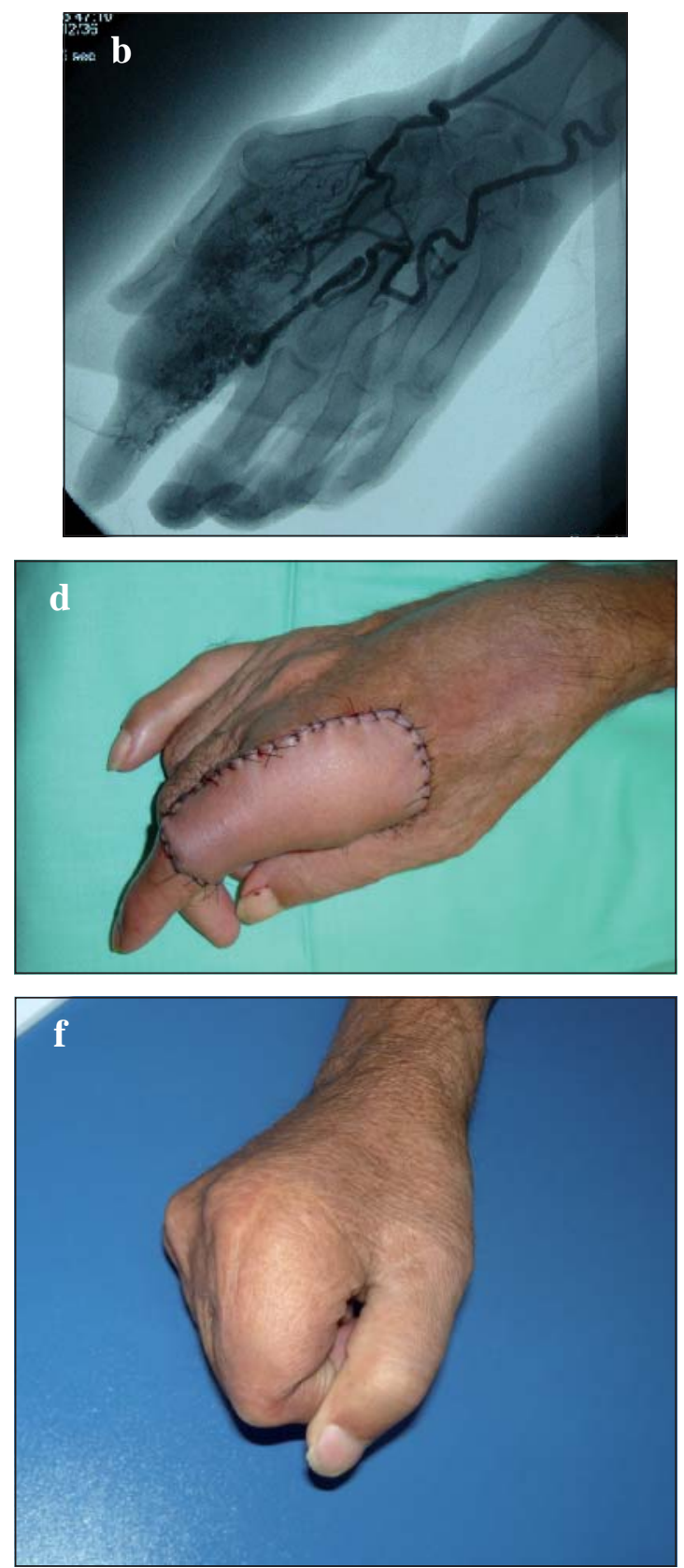
en hueso de la zona dadora; así como fijación del hueso transferido a la zona receptora mediante placas de osteosíntesis moldeadas al contorno del maxilar inferior. Evoluciona favorablemente desde el punto de vista quirúrgico, con recuperación de la deglución y fonación adecuada, con buena perfusión del colgajo, sin complicaciones a los 7 meses del postoperatorio, actualmente bajo tratamiento quimio y radioterápico por patología oncológica de base (Figura 4).
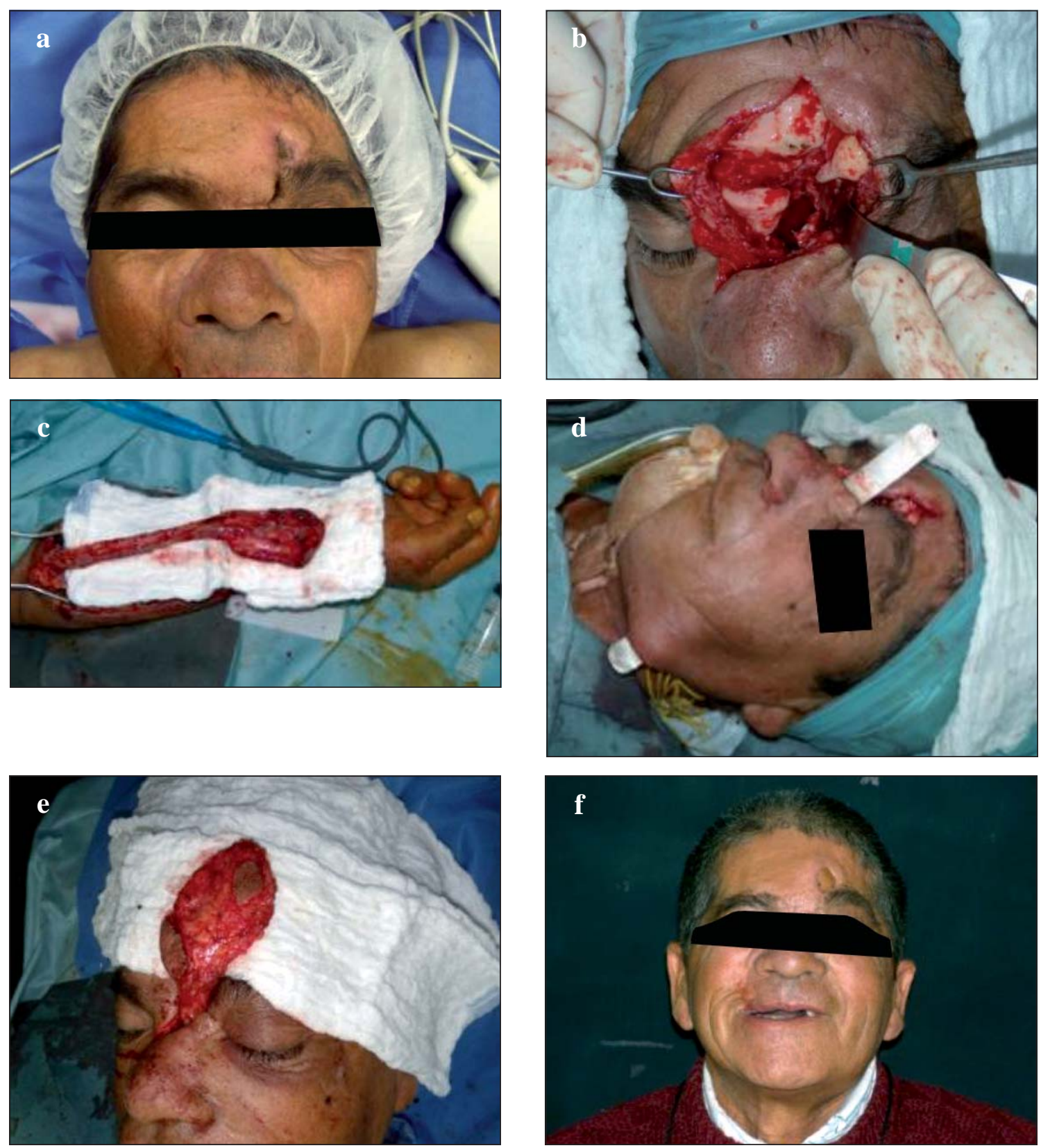

Figura 3. a) fístula cráneo-naso-oftalmocutánea 10 años después del accidente; b) reapertura de la lesión, aseo y retiro de injertos óseos; c) colgajo radial en zona dadora; d) trayecto para anastomosar el pedículo del colgajo radial a la arteria facial; e) colgajo radial posterior a anastomosis microquirúrgica ya en zona receptora y f) control postoperatorio un año después de la reconstrucción. 

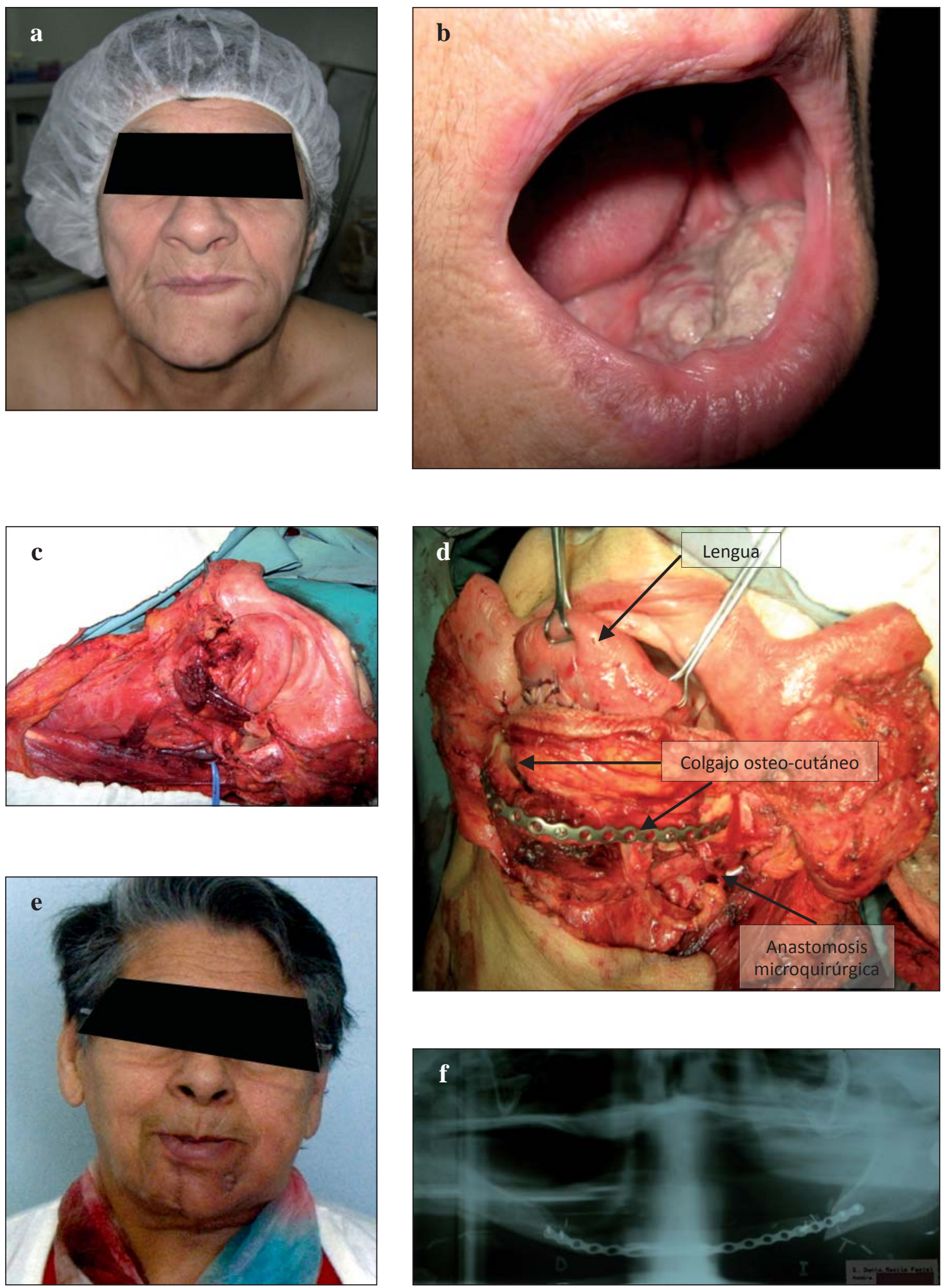

Figura 4. a y b) preoperatorio; c) defecto post resección oncológica; d) reconstrucción en intraoperatorio; e y f) controles postoperatorios a los 6 meses. 


\section{Discusión}

El colgajo radial ha sido desde su aparición una gran herramienta de ayuda en la cirugía reconstructiva, ha logrado mantener su validez a lo largo del tiempo y después de 30 años de su creación, sigue siendo una de las alternativas de cobertura más multifacéticas disponibles. Hemos podido aprender de sus fortalezas y mejorar sus debilidades; destacando entre las ventajas más importantes el hecho de que nos permite utilizar piel, músculos, tendones, tejido adiposo, hueso y nervio según necesidad ${ }^{8}$, aportando piel fina, sin pelo, de color y textura similares a la piel de la cara y el cuello ${ }^{9}$. El componente cutáneo del colgajo es ideal para la cobertura de mucosas, ya que con el tiempo termina modificando su epitelio hasta convertirse en mucosa oral y/o nasal ${ }^{10-13}$, se caracteriza también por ser maleable, flexible y resistente en el tiempo ${ }^{14}$, no tiene contraindicaciones de edad o patologías asociadas ${ }^{15}$ y la zona dadora mantiene su funcionalidad con un acotado compromiso estético y de baja morbilidad. La toma del colgajo es una técnica relativamente simple, se obtiene un "largo" pedículo vascular, de buen diámetro, que permite transferir tejidos a mayor distancia de la zona dadora, sin necesidad de microcirugía ${ }^{2}$.

Como estudio preoperatorio de factibilidad sólo se utiliza el Test de Allen², que es un examen clínico realizable al pie de la cama del paciente; consiste en realizar una isquemia de la extremidad a ser estudiada y así evaluar la perfusión de la mano por sus distintos afluentes en forma separada: con el brazo en alto se debe cerrar y abrir la mano para disminuir el retorno venoso, luego se comprime a nivel de la muñeca el trayecto de las arterias radial y cubital, nuevamente se realiza el cierre y apertura repetido de la mano hasta que se torne pálida y entonces se descomprime el trayecto de la arteria cubital y se evalúa el tiempo en que se logra un adecuado llene capilar de la palma de la mano; si éste es menor a 7 segundos el Test de Allen es positivo (indicando que la perfusión de la mano sí es la adecuada sólo con el flujo de la arteria cubital); si el tiempo de llenado es entre 7-10 segundos, el resultado es dudoso, y si el tiempo supera los 10 segundos, el resultado es negativo (indica que no se logra una adecuada perfusión distal sólo con el aporte de una de las arterias y que necesita de ambas).

Sin embargo, pese a los grandes logros obtenidos a lo largo de su historia, el colgajo radial ha presentado ciertas desventajas importantes de tener en cuenta a la hora de realizarlo, pero que siendo corregidas adecuadamente permiten que éste siga siendo una gran alternativa de reconstrucción. Uno de los principales puntos en contra son las complicaciones que afectan a la "zona dadora" como la dehiscencia de sutura, la exposición de tendón, el requerir curaciones prolongadas o el déficit estético $^{16,17}$. En algunos casos, cuando no se logra un cierre primario (lesiones $>5 \mathrm{~cm}$ ), se debe tomar un IDE para cubrir a la zona dadora o realizar colgajos de avance, de rotación, Z plastías, en V-Y o simplemente implementar los sustitutos dérmicos. No hay que olvidar de que este tipo de colgajo debe inevitablemente sacrificar una arteria importante y que en los casos de realizar el colgajo en un paciente con un Test de Allen no categórico, nos exponemos a una isquemia de la extremidad; el examen no es $100 \%$ específico en todos los pacientes, por lo que ante la duda se debe certificar la permeabilidad del pedículo mediante un estudio doppler de la extremidad y en casos puntuales potencialmente una arteriografía ${ }^{2,18}$. El colgajo osteocutáneo nos da un limitado aporte óseo $(10 \mathrm{~cm})$, permite utilizar sólo una capa de hueso cortical que no tiene la fortaleza suficiente para colocar injertos "osteointegrados" en los casos de reconstrucción maxilar. La "zona dadora” puede verse afectada a largo plazo por fracturas del hueso donante $^{17}$ y es por eso que para reducir el riesgo de movimiento la osteotomía debe ser $<40 \%$ de la circunferencia del hueso radial y ser cortada en bisel $^{6}$, se sugiere preservar la fascia, cubrir tendones, realizar la osteotomía en quilla y colocar una osteosíntesis profiláctica con inmovilización prolongada de la extremidad ${ }^{12,17}$.

Como hemos podido ver, los beneficios de la utilización de este tipo de colgajo, han superado con creces las dificultades de su utilización; no sólo por la plasticidad de sus tejidos, adaptables a distintas zonas receptoras, lo que le ha valido ser el colgajo de elección para la reconstrucción de cabeza, cuello y extremidad superior; sino también por la seguridad con que este procedimiento resuelve en forma definitiva las lesiones de los pacientes atendidos, manteniendo la vigencia absoluta del colgajo radial.

\section{Referencias}

1. Yang GF, Chen PJ, Gao YZ, Liu XY, Li J, Jiang SX, et al. Forearm free skin flap transplantation: a report of 56 cases. 1981 Br J Plast Surg. 1997;50:162-5.

2. Megerle K, Sauerbier M, Germann G. The Evolution of the Pedicled Radial Forearm Flap. Hand 2010;5:37-42.

3. Saint-Cyr M, Mujadzic M, Wong I, Hatef D, Lajoie A. S, Rohrich RJ, et al. The Radial Artery Pedicle Perforator Flap: Vascular Analysis and Clinical Implications. Plast Reconstr Surg. 2010;125:1469-78.

4. Weinzweig N, Chen L, Chen ZW. The distally based radial forearm fasciosubcutaneous flap with preservation of the radial artery: an anatomic and clinical approach. Plast Reconstr Surg. 1994;94:675-84.

5. Lin JY, Cheng MH, Wei FC, Song D, HuangWC. Proxi- 
mal forearm flap based on a septocutaneous vessel from the radial artery. Plast Reconstr Surg. 2006;117:955-60.

6. Weinzweig N, Jones NF, Shestak KC, Moon HK, Davies BW. Oromandibular reconstruction using a keel-shaped modification of the radial forearm osteocutaneous flap. Ann Plast Surg. 1994;33:359-69.

7. Nunez VA, Pike J, Avery C, Rosson JW, Johnson P. Prophylactic plating of the donor site of osteocutaneous radial forearm flaps. Br J Oral Maxillofac Surg. 1999;37:210-2.

8. Omokawa S, Mizumoto S, Fukui A, Inada Y, Tamai S, et al. Innervated radial thenar flap combined with radial forearm flap transfer for thumb reconstruction. Plast Reconstr Surg. 2001;107:152-4.

9. Kao HK, Chang KP, Wei FC, Cheng MH. Comparison of the medial sural artery perforator flap with the radial forearm flap for head and neck reconstructions. Plast Reconstr Surg. 2009;124:1125-32.

10. Andrades P, Rosenthal EL, Carroll WR, Baranano C. F, Peters GE. Zygomatic-maxillary buttress reconstruction of midface defects with the osteocutaneous radial forearm free flap. Head Neck 2008;30:1295-302.

11. Menick FJ, Salibian A. Microvascular repair of heminasal, subtotal, and total nasal defects with a folded radial forearm flap and a full-thickness forehead flap. Plast Reconstr Surg. 2011;127:637-51.
12. Thoma A, Khadaroo R, Grigenas O, Archibald S, Jackson S, Young JE, et al. Oromandibular reconstruction with the radial-forearm osteocutaneous flap: experience with 60 consecutive cases. Plast Reconstr Surg. 1999;104:368-78.

13. Davidson J, Boyd B, Gullane P, Rotstein L, Freeman, J, Manktelow R, et al. Comparison of the results following oromandibular reconstruction using a radial forearm flap with either radial bone or a reconstruction plate. Plast Reconstr Surg. 1991;88:201-8.

14. Thoma A, Levis C, Young JE. Oromandibular reconstruction after cancer resection. Clin Plast Surg. 2005;32:361-75.

15. Talbi M, Stussi JD, Schwenk D, Meley M. Successful radial forearm free flap for facial reconstruction in a 97-year-old patient. Plast Reconstr Surg. 2000;106:1656-7.

16. Avery CM. Review of the radial free flap: is it still evolving, or is it facing extinction? Part one: soft-tissue radial flap. Br J Oral Maxillofac Surg. 2010;48:245-52.

17. Avery CM. Review of the radial free flap: still evolving or facing extinction? Part two: osteocutaneous radial free flap. Br J Oral Maxillofac Surg. 2010;48:253-60.

18. Kajikawa A, Ueda K, Mochizuki Y, Katsuragi Y. An objective Allen test using color Doppler echo. Plast Reconstr Surg. 2010;125:82e-85e. 